\title{
Arbitrarily Accurate Eigenvalues for One-Dimensional Polynomial Potentials
}

\author{
Y. Meurice \\ Department of Physics and Astronomy, The University of Iowa, Iowa City, Iowa 52242, USA
}

\begin{abstract}
We show that the Riccati form of the one-dimensional Schrödinger equation can be reformulated in terms of two linear equations depending on an arbitrary function $G$. When $G$ and the potential (as for anharmonic oscillators) are polynomials the solutions of these two equations are entire functions ( $L$ and $K$ ) and the zeroes of $K$ are identical to those of the wave function. Requiring such a zero at a large but finite value of the argument yields the low energy eigenstates with exponentially small errors. Approximate formulas for these errors are provided. We explain how to chose $G$ in order improve dramatically the numerical treatment. The method yields many significant digits with modest computer means. We discuss the extension of this method in the case of several variables.
\end{abstract}

PACS: 03.65.Ge, 03.65.-w, 02.30.Em, 02.30.Mv, 10.10.St, 33.20.-t

\section{INTRODUCTION}

Quantum anharmonic oscillators appear in a wide variety of problems in molecular, nuclear or condensed matter physics. Typically, anharmonic terms appear in expansions about a minimum of a potential, when ones tries to incorporate the non-linear features of the forces responsible for this equilibrium. The most celebrated example is the quartic anharmonic oscillator [1] where a $\lambda x^{4}$ term is added to the usual harmonic Hamiltonian. Introducing bilinear couplings among a set of such oscillators leads to a rich spectrum, for instance, multiphonon bound states in one-dimensional lattice models [2]. More generally, one can think about the $\lambda \phi^{4}$ (or higher powers of $\phi$ ) field theories in various dimensions as systems of coupled anharmonic oscillators.

Anharmonic terms can be treated perturbatively and the perturbative series can be represented by Feynman diagrams. Unfortunately, the coefficients of the series $[1,3]$ have a factorial growth and the numerical values obtained from the truncated series have an accuracy which is subject to limitations. At fixed coupling, there is an order at which an optimal accuracy is reached. At fixed order, there is a value of the coupling beyond which the numerical values are meaningless even as an order of magnitude. In the case of the single-well quartic potential, Padé approximants can be used for the series or its Borel transform. Rigorous proofs of convergence can be established in particular cases [4]. Unfortunately, such a method does not apply to the case of the double-well potential [5] where instanton effects [6,7] need to be taken into account. It should also be noted that even when Padé approximants converge, the convergence rate may be slow. Strong coupling expansions [8] or variational interpolations [9] sometimes provide more accurate results.

The above discussion shows that finding an expansion which can be used indiscriminately for most quantum mechanical problems with polynomial potentials remains a challenging problem. Alternatively, one can use numerical methods. Variational methods are often used to obtain upper and lower bounds on energy levels $[10,11]$.
These methods are based on rigorous inequalities and are considered superior to methods based on numerical integration [11]. However, the difference between the bounds widens rapidly with the anharmonic coupling and the energy level. Methods based on series expansions in the position variable [12-15] appear to produce more significant digits more easily. However, our understanding of the convergence and numerical stability of these methods seems to be limited to empirical observations. The methods based on series expansions fall into two categories: methods based on the evaluations of determinants $[12,14]$ and methods based on boundary conditions at large but finite values of the position $[13,15]$. The main goal of this article is to provide a systematic discussion of the errors associated with this second category of methods and to show how to make these errors arbitrarily small in the most efficient way. With the exception of Section IX, we only consider one-dimensional problems. We discuss two types of errors. First, the numerical errors made in calculating the energy which makes the wave function vanish at some large value of the position $x_{\max }$. Second, the intrinsic error due to the finiteness of $x_{\max }$.

The basic elements the numerical method used hereafter were sketched in Ref. [15] and applied to the quartic anharmonic oscillator. We wrote the logarithmic derivative of the wave function which appears in the Riccati equation as $L / K$ and showed that these functions were entire. The values of the first ten eigenvalues with 30 significant digits provided for a particular coupling have been used to test new theoretical methods [16]. Two issues were left open in this formulation: first, the basic equations had an interesting invariance which was not undestood but could be used to improve the numerical efficiency; second, the use of the method for parity noninvariant potentials appeared to be unduly complicated [17].

In Section II, we present a new formulation where these two issues are settled. The basic equations presented depend on an arbitrary function denoted $G(x)$. This freedom can be interpreted as a local gauge invariance associated with the fact that only $L / K$ is physical. The wave function is invariant under these local transforma- 
tions. In section III, we show how to construct power series for $L$ and $K$. The complications in the case of parity non-invariant potentials (such as asymmetric doublewells) are minimal. When the potential and the gauge function are polynomials, these series define entire function. In other words, it is always possible to construct arbitrarily accurate solutions of the Schrödinger equation for arbitrary $E$ within a given range of the position variable, by calculating enough terms in the expansions of $L$ and $K$. This allows us to reproduce the asymptotic behavior of the wave function and determine the energy eigenvalues. In section IV, we use the global properties of the flows of the Riccati equation to recall of some basic results related to the WKB approximation and the Sturm-Liouville theorem. We explain how bifurcations in the asymptotic behavior of the functions $K$ and $L$ can be exploited to determine the eigenvalues.

It should be noted that the importance of reproducing the proper asymptotic behavior has been emphasized in variational approaches [18]. It should also be noted that Padé approximants have been used in conjunction with the Riccati equation in Ref. [14], where the quantization condition used was that the approximants give one additional coefficient in the Taylor expansion. This procedure depends only on the coefficients of the expansions used and there is no reference to any particular value of $x$ (as our $\left.x_{\max }\right)$. Consequently, there is no obvious connection between the two approaches.

In the next two sections, we show how to turn the gauge invariance to our advantage. In Section $\mathrm{V}$, the quantitative aspects of the bifurcation are discussed with an exponential parametrization similar to the one used to determine Lyapounov exponents in the study of chaotic dynamical system. The exponents are $G$-dependent. We provide an approximate way to determine the exponents and the energy resolution. We explain how our freedom in chosing $G$ can be used to make the bifurcation more violent and improve the energy resolution. However, the choice of $G$ also affects the convergence of $L$ and $K$ and consequently the numerical accuracy of the solution of the Schrödinger equation. In Section VI, we show in a particular example that for an expansion of $L$ and $K$ at a given order, a judicious choice of gauge can improve tremendously the numerical accuracy of an energy level. We discuss the two principles which allow to make optimal choices of $G$ and provide practical methods to determine approximately this optimal choice for the general case. We use these methods to explain some empirical results found in [13].

In Section VII, we discuss the the error $\delta E$ on the energy levels due to the finiteness of $x_{\max }$. We propose two approximate formulas valid, respectively, for intermediate and large values of $x_{\max }$ and compatible in overlapping ranges. Note that one can reinterpret the condition that the wavefunction vanishes at $x_{\max }$ as coming from a slightly different problem where the potential becomes infinite at $x_{\max }$. In the path-integral formulation (which can be extended immediately to field theory prob- lems), the fact that the potential becomes infinite at $x_{\max }$ means that paths with values of $x$ larger than $x_{\max }$ are not taken into account. It has been argued $[19,20]$ that these configurations are responsible for the asymptotic behavior of the regular perturbative series. In Ref. [20], we showed that the perturbative series of several modified problem were convergent. The error formula sets the accuracy limitations of this approach. Some of the methods used in this section could be used for quantum field theory problems.

The anharmonic oscillator can be considered as a field theory with one time and zero space dimensions. It can be used to test approximate methods such as perturbative expansions or semi-classical procedures. An illustrative example is given in Ref. [23] where multi-instanton effects were considered and where the splitting of the two lowest levels of a double-well problem were estimated with more than hundred digits. In Section VIII, we show that our method can be used to reproduce all these digits. Finally, we discuss the generalization of the method to problems with several variables in Section IX. For these problems, our ability to reduce the degree of expansion by using optimal gauge functions may be crucial.

\section{BASIC EQUATIONS AND THEIR GAUGE-INVARIANCE}

We consider a one-dimensional, time-independent Schrödinger equation $H \Psi=E \Psi$, for an Hamiltonian

$$
H=\frac{p^{2}}{2 m}+\sum_{l=1}^{2 l} V_{j} x^{j} .
$$

As is well-known, one can reexpress the wave function in terms of its logarithmic derivative

$$
\Psi(x) \propto \mathrm{e}^{-\frac{1}{\hbar} \int_{x_{0}}^{x} d y \phi(y)}
$$

and obtain the Riccati form of the equation:

$$
\hbar \phi^{\prime}=\phi^{2}+2 m(E-V) .
$$

It is assumed that $m>0$ and that the leading power of $V$ is even with a positive coefficient $\left(V_{2 l}>0\right)$.

Writing $\phi=L / K$, we obtain a solution of Eq

provided that we solve the system of equations:

$$
\begin{aligned}
& \hbar L^{\prime}+2 m(V-E) K+G L=0 \\
& \hbar K^{\prime}+L+G K=0
\end{aligned}
$$

where $G(x)$ is an unspecified function. This can be seen by multiplying (4) by $K,(5)$ by $L$ and eliminating $G K L$ by taking the difference. One then obtains the Riccati equation (3) multiplied by $K^{2}$. Near a zero of $K$, one can check that Eqs. (4-5) remain valid, namely they impose that $\phi$ has a simple pole with residue $-\hbar$. This 
allows the wave function to become zero and change sign as the contour goes around the pole on either side.

Eqs. (4-5) are invariant under the local transformation

$$
\begin{aligned}
L(x) & \rightarrow Q(x) L(x) \\
K(x) & \rightarrow Q(x) K(x) \\
G(x) & \rightarrow G(x)-\hbar Q^{\prime}(x) / Q(x),
\end{aligned}
$$

where $Q(x)$ is an arbitrary function. It is clear that this transformation leaves $\phi$ and the wave function unchanged. If we choose $G=0$ and eliminate $L$ using Eq. (5), we recover the Schrödinger equation for $K$. Starting from this gauge and making an arbitrary transformation, we find that in general

$$
K(x) \propto \Psi(x) \mathrm{e}^{-\frac{1}{\hbar} \int_{x_{0}}^{x} d y G(y)}
$$

This shows that when $G$ is polynomial, $K$ is simply $\Psi$ multiplied by an entire function with no zeroes [21]. This means that the zeroes of $K$ and $\Psi$ are identical. In other words, there are no spurious zeroes when $G$ is polynomial.

By taking the derivative of Eqs. (4) and (5) and choosing $G(x)$ appropriately, one can obtain the basic Equations used in [15]. The explicit form of $G(x)$ is reached by comparing the two sets of equations and integrating one of the differences. The two possibilities are compatible. The resulting integral expression can be worked out easily by the interested reader. The only important point is that the $G$ found that way is in general not polynomial, justifying the spurious zeroes found with the original formulation.

\section{SOLUTIONS IN TERMS OF ENTIRE FUNCTIONS}

The function $G$ can be chosen at our convenience. For instance, we could impose the condition $K=1$ by taking $G=-L$ and recover the Riccati equation for $L$. However, the main advantage of Eqs. (4-5) is that they are linear first order differential equations with variables coefficients. It is well-known [22] that if we consider these equations for complex $x$, the solutions inherit the domain of analyticity of the coefficients (provided that this domain is simply connected). If the coefficients are entire functions, there exists a unique entire solution corresponding to a particular set of initial values. In the following, we restrict ourselves to the case where $V$ and $G$ are polynomials.

One can construct the unique solution corresponding to a particular choice of initial values $L(0)$ and $K(0)$ by series expansions. Using $K(x)=\sum_{n=0}^{\infty} K_{n} x^{n}$ and similar notations for the other functions, one obtains the simple recursion

$$
L_{n+1}=\frac{-1}{\hbar(n+1)}\left(\sum_{l+p=n}\left(2 m V_{l} K_{p}+L_{l} G_{p}\right)-2 m E K_{n}\right)
$$

$$
K_{n+1}=\frac{-1}{\hbar(n+1)}\left(L_{n}+\sum_{l+p=n} K_{l} G_{p}\right)
$$

Given $L_{0}$ and $K_{0}$, these equations allow to determine all the other coefficients. For potentials which are parity invariant, and if $G$ is an odd function, $L$ and $K$ can be assigned definite and opposite parities. In this case, we can impose the initial conditions $K_{0}=1$ and $L_{0}=0$ for even wave functions and $K_{0}=0$ and $L_{0}=1$ for odd wave functions. If the Hamiltonian has no special symmetry, as for instance in the case of an asymmetric double-well, one could leave $L_{0}$ indeterminate and fix it at the same time as $E$ using conditions on the wave function or its derivative at two different points. These two conditions translate (in good approximation) into two polynomial equations in $L_{0}$ and $E$ and can be solved by Newton's method.

The fact that Eqs. (8) determines entire functions provided that $V$ and $G$ are polynomials can be inferred directly from the fact that the coefficients will decrease as $(n !)^{-\kappa}$ for some positive power $\kappa$ to be determined and in general depending on the choice of $G$. As we will explain in more detail in Section IV, if the leading term in $V$ is $V_{2 l} x^{2 l}$, one expects from Eq. (3) that for $x$ large enough,

$$
\phi(x) \simeq \pm \sqrt{2 m V_{2 l}} x^{l}
$$

and asymptotically,

$$
\Psi(x) \propto \mathrm{e}^{-\frac{ \pm 1}{(l+1) \hbar} \sqrt{2 m V_{2 l}} x^{l+1}} .
$$

Looking at the general expression for $K$ given in Eq. (7), one sees that $K$ will have the same asymptotic behavior provided that the integral of $G$ grows not faster than $x^{l+1}$. If this is the case, then $\kappa=1 /(l+1)$. This behavior is well observed in empirical series.

Note that if $G$ grows faster than $x^{l}$, the coefficients decay more slowly and the procedure seem to be less efficient. In the following, we will mostly discuss the case $l=2$. If we require that $G$ is an odd polynomial growing not faster than $x^{2}$, this means that $G$ is homogeneous of degree 1.

\section{QUANTIZATION FROM GLOBAL FLOW PROPERTIES}

In this section, we use the global properties of the flows associated with the Riccati equation to rephrase some implications of Sturm-Liouville theorem and to justify the asymptotic behavior given in Eq. (9). The main goal of this section is to provide a simple and intuitive picture of the bifurcation which occurs when the value of $E$ is varied by a small amount above or below an energy eigenvalue. The main results of this section are summarized in Figs. 1 and 2. 
We consider the solutions of Eq. (3) obtained by varying $E$ with fixed initial values. It is convenient to introduce an additional parameter $s$ and to rewrite the original equation as a 2-dimensional ODE with an $s$-independent r.h.s .

$$
\begin{aligned}
\hbar \dot{\phi} & =\phi^{2}+2 m(E-V(x)) \\
\dot{x} & =1,
\end{aligned}
$$

where the dot denotes the derivative with respect to $s$.

The flows in the $(x, \phi)$ plane have some simple global properties that we now proceed to describe. We consider a solution (phase curve) with initial condition $x=x_{0}$ and $\phi=\phi_{0}$ at $s=0$. We assume that for these values the r.h.s of Eq. (11) is > 0. It will become clear later that if such a choice is impossible, a normalizable wave function cannot be constructed. With this assumption, the phase curve starts moving up and right as $s$ increases, possibly going through simple poles with residues $-h$. This situation persists unless the r.h.s. of (11) becomes zero. We call the separating curves defined by a zero for the r.h.s of Eq. (11), $\phi= \pm \sqrt{2 m(V(x)-E)}$, "WKB curves". After a phase curve crosses (horizontally) a $W K B$ curve, it moves right and down. If it crosses the $W K B$ curve again, we can repeat the discussion as at the beginning.

At some point, we reach the "last" $W K B$ curve (i.e., the farthest right). For $x$ large enough, the potential is dominated by its largest power and the upper (lower) part of this last WKB curve has a strictly positive (negative) slope. For such values of $x$, if a phase curve crosses the WKB curve, it will do so horizontally and move inside the region where the r.h.s. of Eq. (11) is negative. As $s$ further increases, $\phi$ decreases, but the phase curve cannot cross horizontally the lower part of the WKB curve which has a strictly negative slope. In the same region, if $\phi$ has a pole, the curve reappears below the lower part of the WKB curve and will never take positive values again.

In summary, if in the region described above, a phase curve crosses the WKB curve or develops a pole, then it cannot develop a pole again. The other logical possibility is that the phase curve does none of the above. It is thus clear that for fixed $E$, we can always find a $X$ such that if $x>X, \phi(x)$ has no pole. Consequently the two terms involving $\phi$ in Eq. (3) cannot grow faster than $2 m(E-V)$. Otherwise, $2 m(E-V)$ would become negligible and a pole would be necessary. At least one of these two terms needs to match $2 m(E-V)$. Inspection of the two possibilities leads to Eq. (9). Only the positive solution which follows asymptotically the upper WKB curve leads to a normalizable wave function.

If we compare two phase curves with identical initial conditions but different $E$, the one with larger $E$ initially lays above the other one. If the one with lower $E$ has a first pole at $x_{1}$, then the one with larger $E$ has a first pole at some $x<x_{1}$. Remembering that the poles of $\phi$ produce zeroes of $\Psi$, this rephrases the main idea behind the Sturm-Liouville theorem. An exact energy eigenstate $E_{n}$ is obtained when the wave function has its last zero at infinity. When $E$ is fine-tuned to that value, $\phi$ follows closely the upper branch of the WKB curve. This trajectory in unstable under small changes in $E$. If the energy is slightly increased with respect to $E_{n}, \phi$ develops a pole and reappears on the lower part of the WKB curve. If the energy is slightly decreased with respect to $E_{n}, \phi$ crosses the upper part of the WKB curve and reaches the lower part of the WKB curve. This is illustrated in Fig. 1 in the case of the ground state of the quartic single-well anharmonic oscillator with $m=1 / 2, \hbar=1, V_{2}=1$ and $V_{4}=0.1$. All the figures in this section and the next two sections have been done with this particular example.

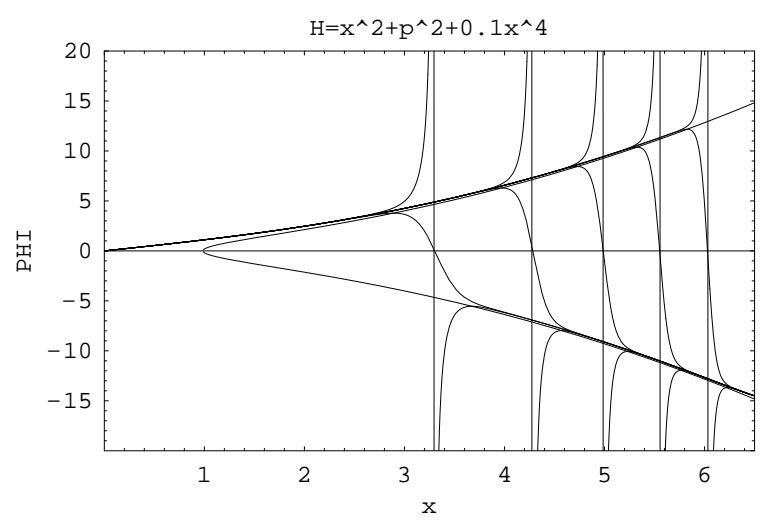

FIG. 1. Bifurcations of $\phi(x)$ from the upper part of the WKB curve associated with the ground state energy $E_{0}$ for energies $E_{0} \pm 10^{-5}, E_{0} \pm 10^{-10}, E_{0} \pm 10^{-15}, E_{0} \pm 10^{-20}$ and $E_{0} \pm 10^{-25}$ (from left to right).

The sensitive dependence on $E$ is also present in the asymptotic behavior of $K$. If the energy is slightly increased with respect to $E_{n}, K$ reaches zero at a finite value of $x$. If the energy is slightly decreased with respect to $E_{n}, K$ increases rapidly. This is illustrated in Fig. 2 for the same example as in Fig. 1.

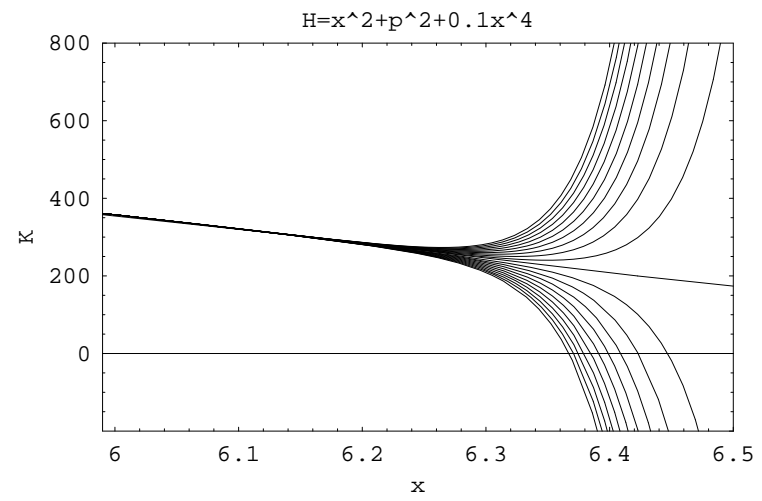

FIG. 2. Bifurcations of $K(x)$ from its trajectory for $E=E_{0}$. The changes in $E$ are $\pm 10^{-30}, \pm 2 \times 10^{-30}, \ldots$, $\pm 10^{-29}$

We now discuss the initial value $\phi_{0}$. For parity invariant potentials, one only needs to consider the cases $\phi_{0}=0($ even $\Psi)$ or $\phi_{0}=-\infty($ odd $\Psi)$ at $x_{0}=0$. For po- 
tentials with no reflection symmetry, one needs to insure that the appropriate behavior is reached when $x \rightarrow-\infty$. This can be implemented in good approximation by requiring that the wave function has also a zero at some large negative value $x_{\text {min }}$. For potentials with a reflection symmetry about another point $x_{1}$ than the origin, one can impose that the wave function $\left(K\left(x_{1}\right)=0\right)$ or its derivative $\left(L\left(x_{1}\right)=0\right)$ vanish at that point. In all cases, we have an independent condition which allows to determine $\phi_{0}$.

In summary, for $x_{\max }$ large enough, the condition

$$
K\left(x_{\max }\right)=0
$$

provides sharp upper bound on the energy levels. The lower part of Fig. 2 makes clear that as $x_{\max }$ increases, sharper bounds are reached. For potentials that are not parity invariant, an additional condition has to be imposed. In all cases, one obtains polynomial equations which can be solved for the energy levels given the potential or vice-versa using Newton's method. Note also that a sharp lower bound can be found by solving $L\left(x_{\max }\right)=0$. The fact that in Fig. 2, a zero of $K$ at $E_{0}+\delta$ corresponds to a zero of $L$ at $E_{0}-\delta$, suggests that the exact value should be very close to the average of the two bounds.

\section{V. $G$-DEPENDENCE OF THE BIFURCATION}

The strength of the bifurcation in $K$ illustrated in Fig. 2 can be approximately characterized by local exponents. If we consider the departure $\delta K(x)$ from $K(x)$ calculated at some exact energy level $E_{n}$, we expect the approximate behavior:

$$
\delta K(x) \simeq C\left(E-E_{n}\right) \mathrm{e}^{x B} .
$$

In other words $\ln (|\delta K(x)|)$ is linear with a slope $B$ independent of the choice of $E$ and an intercept that varies like $\ln \left(\left|E-E_{n}\right|\right)$. This situation is approximately realized in the example considered before as shown in Fig. 3. We have checked in the same example that the sign of the energy difference plays no role. In other words, the same values of $C$ and $B$ can be used above and below $E_{n}$.

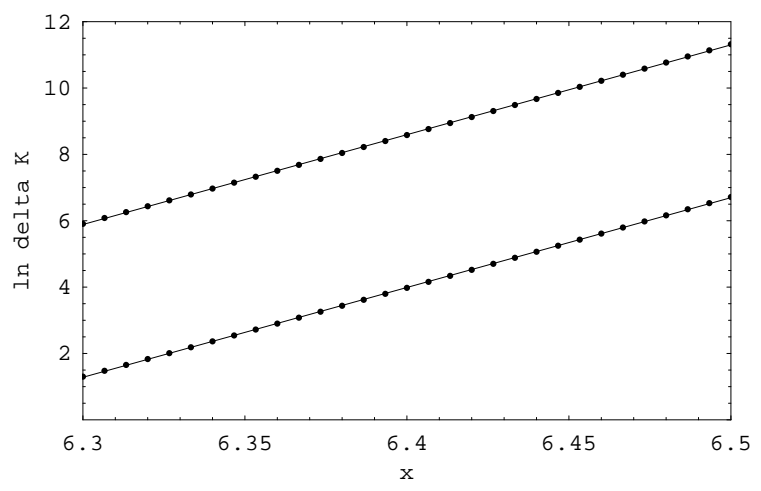

FIG. 3. Natural logarithm of $\delta K(x)$ for $E-E_{0}=10^{-30}$ (lower set of point) and $E-E_{0}=10^{-28}$ (upper set of point). Lines are linear fits.

The exponent $B$ is not uniform. It increases with $x$ and is $G$-dependent as shown in Fig. 4. The local values of $B$ have been calculated by fits in regions of width 0.2 with central value displayed in the horizontal label of Fig. 4.

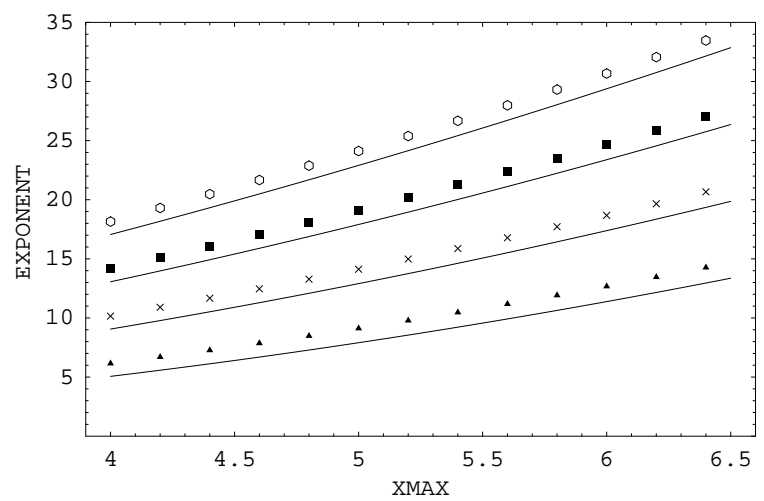

FIG. 4. Value of $B$ for various $x$ and for $G=-3 x$ (empty hexagons), $G=-2 x$ (filled squares) $G=-x$ (crosses) and $G=0$ (triangles). The continuous lines have been drawn using Eq. (16).

The change in $B$ can be understood as follows. If $E$ is changed from $E_{n}$ to $E_{n}+\delta E$, then at some point we have a sudden transition from the upper to the lower WKB curve and asymptotically

$$
\delta \Psi(x) \propto \delta E \mathrm{e}^{+\frac{1}{(l+1) \hbar} \sqrt{2 m V_{2 l}} x^{l+1}} .
$$

Using Eq. (7) and expanding about $x_{\max }$, we obtain that, in good approximation,

$$
B \simeq \frac{1}{\hbar}\left(\sqrt{2 m V_{2 l}} x_{\max }^{l}-G\left(x_{\max }\right)\right) .
$$

As shown in Fig. 4, this simple expression provides reasonable estimates of $B$. The slight underestimation comes in part from the fact that Eq. (16) does not take into account the harmonic term in $V$. Eq. (16) shows that we can increase the strength of the bifurcation near $x_{\max }$ by increasing $x_{\max }$ or $-G\left(x_{\max }\right)$. This allows us to "resolve" the energy more accurately. However, at the same time our numerical resolution of $K\left(x_{\max }\right)$ is affected and we need to take this effect into account. This question is treated in the next Section. In general, if we can establish that $K\left(x_{\max }\right)$ at an energy $E$ very close to $E_{n}$, can be calculated with some numerical accuracy $\delta K^{n u m}$, we have the approximate numerical energy resolution

$$
\left.\delta E^{n u m} \propto \delta K^{n u m} \cdot \mathrm{e}^{+\frac{1}{\hbar}\left(\frac{-1}{l+1} \sqrt{2 m V_{2 l}} x_{\max }^{l+1}+\int_{0}^{x} \max \right.} d x G(x)\right) .
$$




\section{AN OPTIMAL CHOICE OF $G$}

In this Section, we show that from a numerical point of view, the choice of $G$ is important. We discuss the question of an optimal choice, first with an example and then in general. We start with the calculation of the ground state in the case $m=1 / 2, \hbar=1, V_{2}=1$ and $V_{4}=0.1$. We discuss the estimation of the ground state energy using the equation $K\left(x_{\max }\right)=0$ with $x_{\max }=6$. The fact that we use this finite value for $x_{\max }$ creates an error in the 25-th digit (see Section VII).

From the discussion of Section III, it is reasonable to limit the discussion to a gauge function of the form

$$
G(x)=-a x,
$$

which using Eq. (7) implies that

$$
K(x) \propto \Psi(x) \mathrm{e}^{\frac{1}{2 \hbar} a x^{2}}
$$

With this restriction, the optimization problem is reduced to the determination of $a$. As $a$ increases through positive values, the features of $\Psi$ are exponentially amplified, making the bifurcation displayed in Fig. 2 more violent. Ideally, we would like to take $a$ as large as possible. However, if $a$ is too large, we may need too many coefficients $K_{n}$ to get a good approximation. If we consider the problem at a given order, the two requirements of sensitivity and accuracy result in a compromise which determines the optimal value of $a$.

As explained in Section III, the choice of Eq. guarantees a suppression of the form $(n !)^{\frac{-1}{3}}$ for the coefficients of $L$ and $K$. However, the choice of $a$ still affects significantly the behavior of these coefficients as shown in Fig. 5.

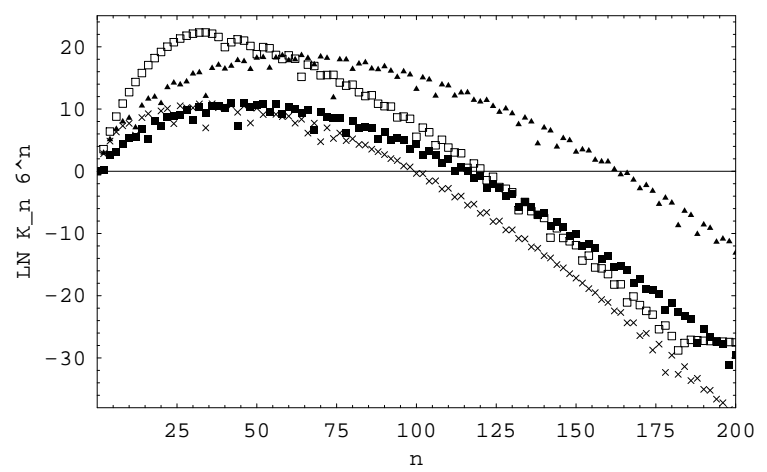

FIG. 5. $\ln \left(\left|K_{n} 6^{n}\right|\right)$ versus $n$, for $G=0$ (triangles), $G=-x$ (filled squares), $G=-2 x$ (crosses) and $G=-3 x$ (empty squares).

The quantity $K_{n} x_{\max }^{n}$ is relevant to decide at which order we need to truncate the series in order to get a good estimate of $K\left(x_{\max }\right)$. For instance, if we require knowing $K\left(x_{\max }\right)$ with errors of order 1 , we need about 100 coefficients for $a=2$ but more than 150 for $a=0$. The corresponding values for $a=1$ and 3 fall between these two values, indicating that $a=2$ is close to optimal. This estimate is confirmed by an analysis of the dependence of $K_{n}$ on $a$. Sample values are shown in Fig. 6 . We observe rapid oscillations (that we will not attempt to explain) and slowly varying amplitudes which have a minimum slightly below 2 . Note that on the logarithmic scale of Fig. 6 , the zeroes of $K_{n}$ give $-\infty$, however due to the discrete sampling of $a$, it just generates isolated dots on the graphs. Note also that in Figs. 5 and 6 , the coefficients have been calculated for an an accurate value of the ground state energy.

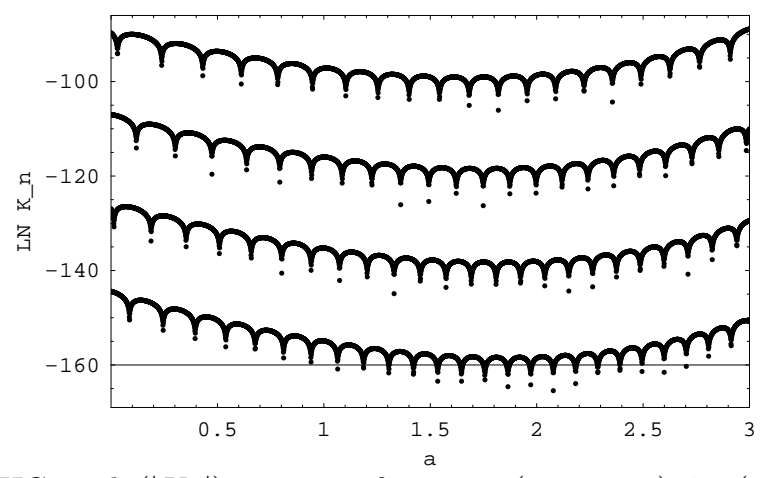

FIG. 6. $\ln \left(\left|K_{n}\right|\right)$ versus $a$, for $n=60$ (upper set), 70 (next set), 80 (next set) and 90 (lower set).

The behavior of the $K_{n}$ calculated at value of $E$ sufficiently close to an eigenvalue, can be understood by using the asymptotic form

$$
K\left(x_{\max }\right) \propto \mathrm{e}^{\frac{1}{\hbar}\left(-\frac{1}{l+1} \sqrt{2 m V_{2 l}} x_{\max }^{l+1}-\int_{0}^{x_{\max }} d x G(x)\right)} .
$$

We emphasize that the relative sign between the two terms in the exponential is opposite than in Eq. (17), because we are now on the upper WKB curve. For $a=0$, Eq. (20) provides a rough estimate of $K_{n} x_{\max }^{n}$. Remembering the minus sign in the parametrization of $G$ (Eq. (18)), we see that if $a$ is given a small positive value, the argument of the exponential in Eq. (20) decreases and we can obtain comparable accuracy with less terms in the expansion. Naively, our optimum choice is obtained when the two terms in the exponential cancel. In the general case, this amounts to having

$$
\sqrt{2 m V_{2 l}} x_{\text {max }}^{l+1} \simeq-(l+1) \int_{0}^{x_{\max }} d x G(x) .
$$

For the particular example considered here, this cancellation is obtained for $a=(2 / 3) \sqrt{0.1} x_{\max } \simeq 1.27$. It is clear that when the two terms cancel, subleading terms neglected in Eq. (9) should be taken into account. However, in several examples, we found that this simple procedure gives results close to what is found empirically.

We now address the more general question of determining the $G$-dependence of the number of significant digits that can be obtained from the condition $K\left(x_{\max }\right)=0$ using an expansion of $K$ truncated at a given order. For the example considered before in this section, we see from 
Fig. 7 that, for instance for a truncation at order 100, the most accurate answer is obtained for $a \simeq 1.6$. It is worth noting that for this value of $a$, one gains more than 15 significant digits compared to the $G=0$ case! This figure also indicates, that as expected, the best possible answer (in the present case, 25 significant digits) can always be achieved by calculating enough coefficients.

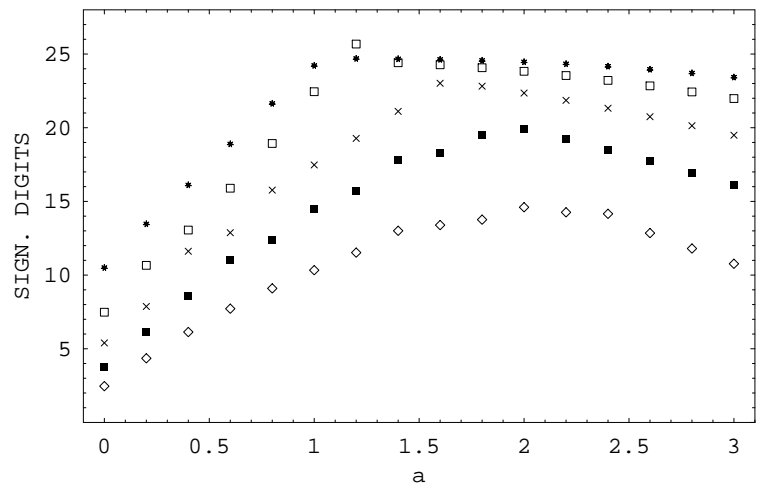

FIG. 7. Number of significant digits for $E_{0}$ versus $a$ using the condition $K(6)=0$ with expansions of order 50 (empty diamonds), 75 (filled squares), 100 (crosses), 125 (empty squares) and 150 (stars).

Using Eq. (17) and Fig. (6), we were able to reproduce approximately the left part of Fig. $7(0<a<1)$. To give a specific example, at order 100 , when one changes $a$ from 0 to $1, \delta K^{\text {num }}$ becomes 4 orders of magnitude smaller and the factor $\mathrm{e}^{-\left(\frac{a}{2 \hbar}\right) x_{\max }^{2}}$ improves the resolution by almost 8 orders of magnitude. This approximately accounts for the gain of 11 significant digits observed in Fig. 7. A detailed understanding of the figure in the region $1<a<2$ is beyond what can be accomplished using the asymptotic form of the wave function. However, the naive estimate of Eq. (21) provides a reasonable estimate of the location of the optimal $a$.

It should be noted that an ansatz of the form of Eq. (19) with $a=1$ has been used in Ref. [12] and that the fact that varying $a$ could improve the numerical efficiency was found empirically in Ref. [13]. Eq. (21) can be used to understand these results. For instance, for $H=p^{2}+x^{2}+x^{8}$, we can obtain a very accurate result with $x_{\max }=2.8$ (see Section VII). According to Eq. (21) the optimal value of $a$ in this case is $a=(2 / 5) x_{\text {max }}^{3} \simeq 8.8$ which is slightly below the value $(\approx 10)$ suggested in [13]. Note also that equivalently good results can be obtained using $G=-b x^{3}$.

\section{APPROXIMATE ERROR FORMULAS}

In this Section,we discuss the intrinsic error $\delta E=$ $E\left(x_{\max }\right)-E(\infty)$ where $E\left(x_{\max }\right)$ is defined by $\psi\left(x_{\max }, E\left(x_{\max }\right)\right)=0$, for a given energy level. We emphasize that $\delta E$ is the error due to the finiteness of $x_{\max }$ independently of practical considerations regard- ing the numerical estimation of $E\left(x_{\max }\right)$ which is assumed to be known with an error much smaller than $\delta E$ in this section. We use the familiar parametrization of the quadratic term of the potential, $V_{2}=\frac{1}{2} m \omega^{2}$ and we restore the dependence on $\hbar$ and $m$. The error for the ground state of the harmonic oscillator has been estimated in Eq. (4) of Ref. [20]. Using the asymptotic form of the integral in this equation, we obtain

$$
\delta E_{0}^{h a r m} \simeq 2\left(\frac{S_{0}}{\pi \hbar}\right)^{\frac{1}{2}} \mathrm{e}^{-S_{0} / \hbar}
$$

with

$$
S_{0}=\int_{-\infty}^{+\infty} d t \frac{1}{2} m\left(\left(\dot{x}_{c}(t)\right)^{2}+\omega^{2}\left(x_{c}(t)\right)^{2}\right)=m \omega x_{\max }^{2}
$$

and $x_{c}(t)=x_{\max } \mathrm{e}^{-\omega\left|t-t_{0}\right|}$. This corresponds to semiclassical approximation where the contribution of the large field configurations are obtained by calculating the quadratic fluctuations with respect to $x_{c}(t)$. The anharmonic corrections can be approximated to lowest order in the the anharmonic couplings by adding a term $S_{a n h}$ to $S_{0}$ in the exponent of Eq. (22) with

$$
S_{a n h}=\int_{-\infty}^{+\infty} d t V_{a n h}\left(x_{c}(t)\right)
$$

and $V_{a n h}$ is the anharmonic part of the potential. Our final perturbative estimate is thus

$$
\delta E_{0} \simeq \delta E_{0}^{h a r m} \cdot \mathrm{e}^{-\sum_{j=2}^{l}\left(\frac{1}{j \hbar}\right) V_{2 j} x_{\max }^{2 j}}
$$

This estimate is accurate if the $V_{2 j}$ and $x_{\max }$ are small enough. We expect that for the excited states, approximate formulas of the form of Eq. (25) multiplied by a polynomial should hold.

When $\lambda$ or $x_{\max }$ become too large, Eq. (25) is not adequate. To obtain a better approximation, we use

$$
\frac{\partial}{\partial x_{\max }} \psi\left(x_{\max }, E\left(x_{\max }\right)\right)=0
$$

and the asymptotic behavior of $\Psi$. We estimate that $\partial \Psi / \partial E$ is of the order of the non-normalizable WKB solution and as a consequence, $\delta E$ has the asymptotic form

$$
\delta E \simeq P\left(x_{\max }\right)\left(\psi\left(x_{\max }\right)\right)^{2}
$$

where $P$ is a polynomial. This form is correct for the ground state of the harmonic oscillator. In the case where the leading term of $V$ is $V_{2 l} x^{2 l}$, this implies the asymptotic order of magnitude estimate

$$
\delta E \approx \mathrm{e}^{-\frac{2}{(l+1) \hbar} \sqrt{2 m V_{2 l}} x_{\max }^{l+1}},
$$

We have tested the two approximate errors formulas given above (Eqs. (25) and (28)) for the ground state corresponding to $V_{a n h}=\lambda x^{4}$. We used the numerical 
values $\hbar=m=\omega=1$ and $\lambda=0.1$ The results are shown in Fig. 8. We see that for small values of $x_{\max }$, the perturbative estimate of Eq. (25) corrects properly the harmonic result. However when $x_{\max }$ increases, the Eq. (28) gives better results. If the left part of the graph is displayed with a log-log scale, it is approximately linear with a slope close to 3 . In Fig. (8), the proportionality constant not given by Eq. (28) has been determined by fitting the 5 last data points on the right of the figure. We conclude that by combining the two approximations it is possible to get a reasonable estimate of the errors on $E$ over a wide range of $x_{\max }$.

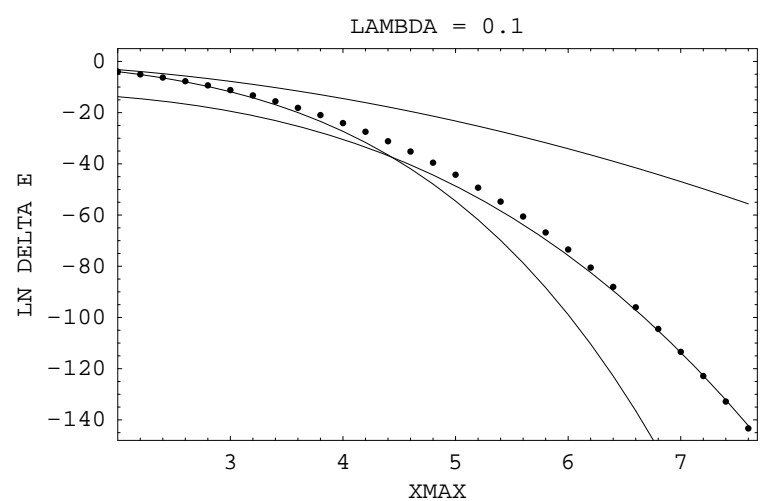

FIG. $8 . \ln \left(\delta E_{0}\right)$ as a function of $x_{\max }$ for $\lambda=0.1$ (black dots). The continuous lines are from top to bottom on the left of the figure: the harmonic case (Eq. (22)), Eq. (25) with $V_{4}=0.1$ (fits the dots well on the left of the figure), Eq. (28) (fits the dots well on the right of the figure).

We have tested Eq. (28) for other potentials. For instance, for $H=p^{2}+x^{2}+x^{8}$, in order to get 30 significant digit, we estimated that $x_{\max } \simeq 2.8$. We found that the difference between the ground state energy found from the condition $K=0$ (upper bound) and $L=0$ (lower bound) differed in the 30th significant digits.

\section{A CHALLENGING TEST}

The only practical limitation of the method proposed here is that in some cases the relevant details of the potential appear in widely separated regions, forcing us to calculate a huge number of coefficients with many significant digits. A simple example where such problem may occur is the symmetric double-well with a small quartic coupling where the separation between the wells goes like the inverse square root of the quartic coupling.

In Ref. [23], the lowest even and odd energies were calculated for a potential with $m=1, \hbar=1, V_{2}=$ $-1 / 4, V_{4}=1 / 2000$ with 180 significant digits. Remarkably, the authors were able to reproduce the 110 significant digits of the splitting between these two states by calculating instanton effects. We have reproduced the 180 digits of both states using an expansion of order 1700 for $K$ and a value of $x_{\max }=46$. The calculations were performed with 700 digit arithmetic. The calculation of one level with such a procedure takes less than two hours with MATHEMATICA on an unexpensive laptop using Pentium3. The computation time increases with the accuracy required. In order to fix the ideas, it takes less than 2 minutes minutes to reproduce the first 120 digits in the above calculation.

\section{THE MULTIVARIABLE CASE}

The basic equations presented in Section II can be extended when the single variable $x$ is replaced by a $N$ dimensional vector $\vec{x}$. In Eq. (2), $\phi$ becomes a vector $\vec{\phi}$ and the integral a line integral. In order to guarantee that the wave function is independent of the choice of the line, we require that the curl of $\vec{\phi}$ vanishes. Eq. (3) becomes:

$$
\hbar \vec{\nabla} \vec{\phi}=\vec{\phi} \cdot \vec{\phi}+2 m(E-V)
$$

Using $\vec{\phi}=\vec{L} / K$, we write as previously

$$
\begin{aligned}
& \hbar \vec{\nabla} \vec{L}+2 m(V-E) K+\vec{G} \cdot \vec{L}=0 \\
& \hbar \vec{\nabla} K+\vec{L}+\vec{G} K=0,
\end{aligned}
$$

with $\vec{G}(\vec{x})$ unspecified at this point. These equations imply the multivariable Riccati equation (29) multiplied by $K^{2}$. Near a zero of $K$, these equations imply the same singularity as Eq. (29). After using Eq. (31), the condition that $\phi$ has no curl reads

$$
\nabla_{i} L_{(j)}+G_{(i)} L_{(j)}=\nabla_{j} L_{i}+G_{(j)} L_{(i)} .
$$

The parenthesis for the vector indices are used in order to distinguish these indices from the order in a power series expansion used later.

The transformation Eqs. (7) can vectorized trivially with $Q$ treated as a scalar. In the expression of $K$ given by Eq. (7), the integral becomes a line integral and we require that $\vec{G}(\vec{x})$ has a vanishing curl. This condition is also necessary to establish that different derivatives acting on $K$ commute.

The choice of coordinates to be used depends on the choice of boundary conditions imposed. If we require $\Psi$ to vanish on a large hypersphere, hyperspherical harmonics should be used. If we require $\Psi$ to vanish on hypercubes (as suggested for lattice problems in Ref. [20]) cartesian coordinates should be used. To fix the ideas, let us consider the case of cartesian coordinates for two variables $x_{1}$ and $x_{2}$ with boundary conditions on a rectangle. We expand $K\left(x_{1}, x_{2}\right)=\sum_{m, n \geq 0} K_{m, n} x_{1}^{m} x_{2}^{n}$ and similar expansions for the two components of $\vec{L}$. The coefficients can be constructed order by order, with the order of $K_{m, n}$ defined as $m+n$. The terms with one derivative yield the higher order terms. For instance, for $K$, we obtain equations providing $K_{m+1, n}$ and $K_{m, n+1}$ in terms of coefficients of higher order just as in Eq. (8). 
A detailed construction shows that if $V\left(x_{1}, x_{2}\right)$ has no special symmetry, we can determine all the coefficient up to a given order $l$ provided that we supply the values of two coefficients at each intermediate order (for instance $\vec{L}_{m, 0}$ for $\left.m \leq l\right)$. These coefficients together with $E$ are fixed by the boundary conditions $K\left(x_{1 \min }, x_{2}\right)=$ $K\left(x_{1 \max }, x_{2}\right)=K\left(x_{1}, x_{2 \min }\right)=K\left(x_{1}, x_{2 \max }\right)=0$. Taking derivatives with respect to the free variables $x_{1}$ and $x_{2}$, and setting these variables to 0 , we obtain an infinite set of conditions. The truncation of this set, together with the truncation of the expansion in the other variable must be studied carefully. If we consider the special case where the problem can be solved by separation of variables, we see that it is important to maintain a uniform accuracy for all the conditions. If all the coefficients have been calculated up to order $l$, this can achieved in the following way. We retain of the order of $l / 2$ derivatives of the four conditions in such a way that we get exactly $2 l+3$ conditions which can be expanded up to an order close to $l / 2$ in the remaining variable. A practical implementation of this program is in progress.

\section{CONCLUSIONS}

In conclusion, we have shown that accurate estimates of the energy levels of arbitrary polynomial potentials bounded from below can be obtained by solving polynomial equations. The fact that the function $L$ and $K$ are entire guarantees that if we calculate enough terms we will gain proper control of the asymptotic behavior of the wave function. Reaching this goal is in general a difficult task which often requires guesswork and analytical continuations (see e.g., Ref. [24]). Here, the convergence of the procedure is guaranteed and the order at which we can terminate the expansion in order to reach a given accuracy can be estimated. In addition, a systematic understanding and control of the errors due to the finite value of $x_{\max }$ has been achieved.

The understanding of the gauge invariance of the basic equations proposed here completely resolves the issues raised from our initial proposal [15]. By varying $G$, from 0 to $-\phi$, we can interpolate between a situation where $K$ is the wave function to another situation where $K=1$ and $L=\phi$. However, for every other choice of $G$, only the ratio $L / K$ has a direct physical meaning. By properly chosing $G$, we can at the same time improve the convergence of $K$ and amplify the bifurcation toward the the non-normalizable behavior.

The extreme accuracy obtained for two widely separated wells indicates that for reasonably complicated potential, the number of terms that needs to be calculated is not prohibitive. We intend to use this method to test analytical results regarding the role of large configurations in the path-integral and to test semi-classical treatment of potentials with asymmetric wells $[6,7]$.

The method can be extended in the case of several vari- ables. It remains to be determined if the simultaneous solution of many polynomial equations can be accomplished with a reasonable accuracy. For these problems, the fact that a judicious choice of the arbitrary functions $\vec{G}$ allows to decrease the order of the expansions may be crucial.

\section{ACKNOWLEDGMENTS}

We thank B. Oktay for communicating his work regarding the treatment of parity non invariant potentials with the method of Ref. [15]. We thank P. Kleiber, W. Klink, L. Li, G. Payne, W. Polyzou, M.H. Reno and V.G.J. Rodgers for valuable conversations. We thank F. Fernandez for pointing out Ref. [13] to us. This research was supported in part by the Department of Energy under Contract No. FG02-91ER40664, and in part by the CIFRE of the University of Iowa.

[1] C. Bender and T. T. Wu, Phys. Rev. 184, 1231 (1969).

[2] W. Z. Wang, J. Tinka Gammel, A. Bishop and M. Salkola, Phys. Rev. Lett, 76, 3598 (1996).

[3] J. C. Le Guillou and J. Zinn-Justin, Large-Order Behavior of Perturbation Theory (North Holland, Amsterdam, 1990) ands Refs. therein.

[4] J. Loeffel, A. Martin, B. Simon, and A. Wightman, Phys. Lett. B 30, 656 (1969); B. Simon, Ann. of Phys. 58, 76 (1970).

[5] E. Brezin, G. Parisi and J. Zinn-Justin, Phys. Rev. D 16, 408 (1977).

[6] S. Coleman, Aspects of Symmetry (Cambridge University Press, Cambridge, 1985).

[7] J. Zinn-Justin, Quantum Field Theory and Critical Phenomena, (Oxford, 1989).

[8] E. Weniger, Phys. Rev. Lett, 77, 2859 (1996); L. Skala, J. Cizek, E. Weniger and J. Zamastil, Phys. Rev. A 59 102 (1999).

[9] H. Kleinert, Phys. Lett. A207, 133 (1995).

[10] N. Bazley and D. Fox, Phys. Rev. 124, 483 (1961); C. Reid, J. Chem. Phys. 43, S186 (1965).

[11] G. Payne and L. Schlessinger, J. Comp. Phys. 13, 266 (1973).

[12] S. Biswas et al. Jour. Math. Phys. 14, 1190 (1973)

[13] J. Killingbeck, Phys. Lett. 84A, 95 (1981).

[14] F. Fernandez, Q. Ma and R. Tipping, Phys. Rev. A 39, 1605 (1989).

[15] B. Bacus, Y. Meurice, and A. Soemadi, J. Phys. A 28, L381 (1995).

[16] F. Antonsen, Phys. Rev. A 60, 812 (1999); W. Bietenholz and T. Struckmann, Int. Jour. Mod. Phys. C 10, 531 (1999).

[17] B. Oktay, unpublished.

[18] A. Turbiner, Sov. Phys. Ups. 27, 668 (1984). 
[19] S. Pernice and G. Oleaga, Phys. Rev. D 57, 1144 (1998).

[20] Y. Meurice, Phys. Rev. Lett. 88, 141601 (2002); Y. Meurice, Nucl. Phys. B (Proc. Suppl.) 106, 908 (2002).

[21] K. Knopp, The Theory of Functions, Part II (Dover, New York, 1975), Ch. 1 section 1.

[22] E. Coddington and N. Levinson, Theory of Ordinary Dif- ferential Equations (Mc Graw-Hill, New York, 1955), Ch. 3 section 7 .

[23] U. Jentschura and J. Zinn-Justin, J. Phys. A 34, L253 (2001).

[24] C. Bender and L. Bettencourt, Phys. Rev. Lett 77, 4114 (1996). 


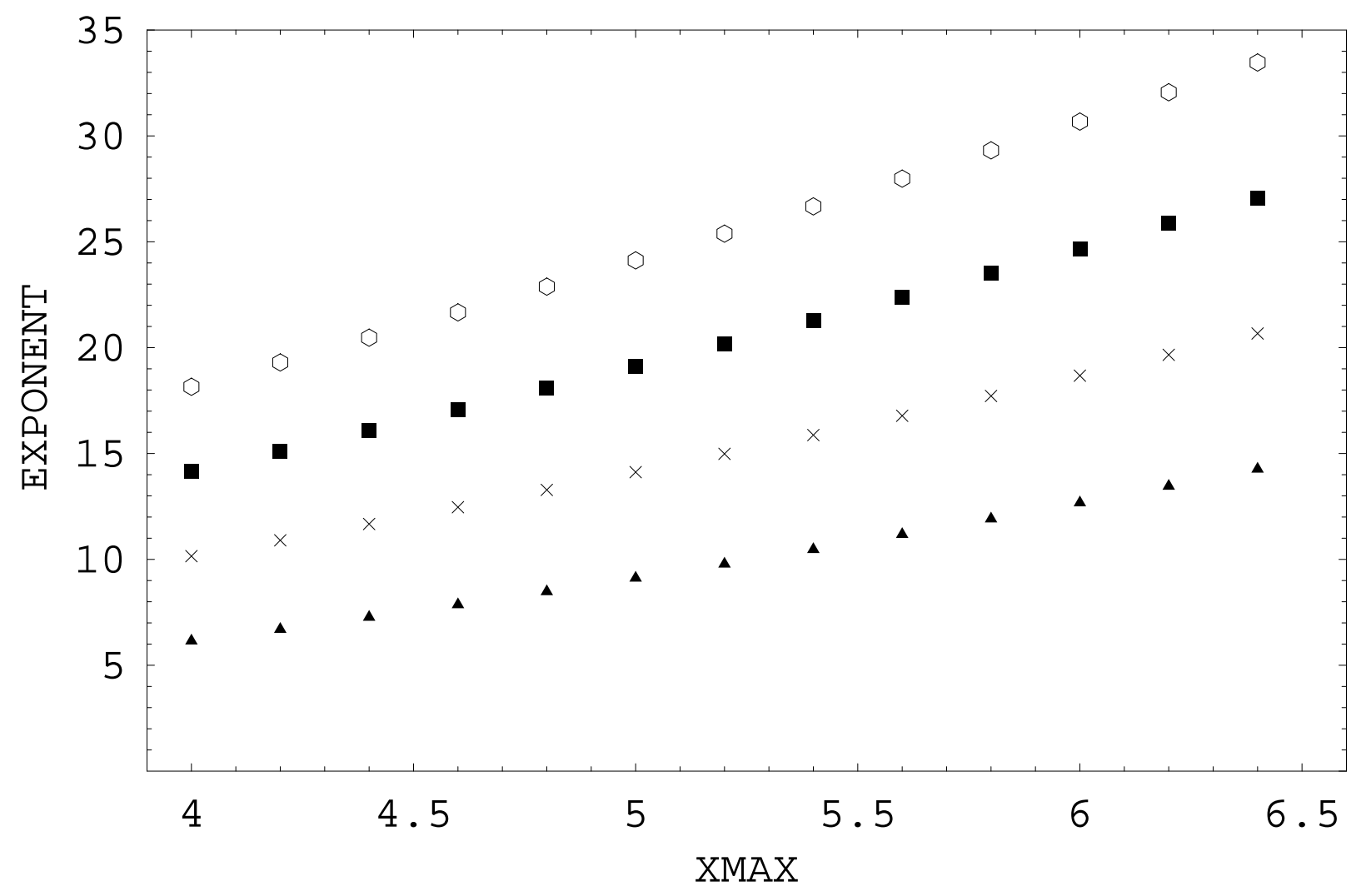

